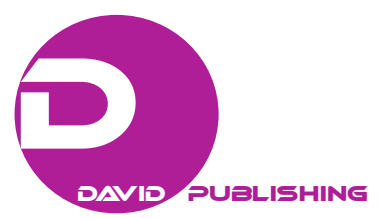

\title{
Georg Cantor’s Ordinals, Absolute Infinity \& Transparent Proof of the Well-Ordering Theorem
}

\author{
Cantor's 1899 Letter to Dedekind
}

\author{
Hermann G. W. Burchard \\ Oklahoma State University, Stillwater OK, USA
}

\begin{abstract}
Georg Cantor's absolute infinity, the paradoxical class $\Omega$ of all ordinals, a non-entity for which being called a "class" is an undeserved dignity, must be the ultimate vexation for mathematical philosophers who hold on to some residual realism in set theory. By careful use of $\Omega$, we can rescue Georg Cantor's 1899 "proof” sketch of the Well-Ordering Theorem (being generous, considering his declining health) by taking the contrapositive of his suggestion and adding Zermelo's choice function, resulting in a concise and uncomplicated proof of the Well-Ordering Theorem.
\end{abstract}

Keywords: Well Ordering Theorem, Burali-Forti’s Paradox, Kant’s Oneness Function

\section{The New Brain Model \& Mathematical Philosophy}

We begin with a discussion of how mathematicians commonly view their profession's validity, reliability, \& veracity, given the high esteem it is granted by the general public. According to the most popular attitude, mathematical entities exist outside ourselves, thought to have been Plato's and therefore called Platonism, although Plato gave no hint acceptable today of the where \& how. Hence most take the Formalist escape, that all that really matters is our mathematical work, how we correctly carry out lemma and theorem formulation, proofs consisting in logical deductions \& calculations, symbol arrangements according to formal rules.

There is good reason for us to think in terms of a new brain model based on functional laterality of the two cerebral hemispheres. This duality has been given high rank by awarding the 2014 Kavli Prize to Brenda Milner, McGill University, for pioneering work by her \& coworkers on the bilateral brain (Milner, 1971; Frisk \& Milner, 1989; Smith \& Milner, 1990). Questions in the research area now are being pursued hotly at several labs. To be brief, let's just agree for the present essay that neuroscience confirms Immanuel Kant's dual rational cognitive capacities \& that these are accounted for by the bilateral brain (Burchard, 2011; H. Burchard \& J. Burchard, 2016):

(A) Left cerebral hemisphere is for Kant's inner sense \& noumena, in time sequentially analyzing memory of the entire past history of the individual, \& making plans, based on sequential logic and verbal language

Hermann G. W. Burchard, Prof. Emer., Dept. of Mathematics, Oklahoma State University, Stillwater OK 74078, USA. 
structures.

(B) Right cerebral hemisphere is for Kant's outer sense \& phenomena, in space perceiving images of scenes in the outer world \& anticipating events as they develop, based on geometry and dynamics.

The time-space duality became apparent when it was found that the hippocampus, an ancient part of the cerebral cortex, does temporal processing on the left side of the brain and allospatial on the right (Igloi, Doeller, Berthoz, Rondi-Reig, \& Burgess, 2010). It is not clear that the Kantian angle has become known to any or all of the workers in the area.

A typical example I like to cite is from neuroscience experiments where subjects are asked to describe the boundaries of imaginary scenes. Using MRI, the authors found activity in the left brain leading them to remark: "The left lateralization of the boundary effect is apparently at odds with neuropsychological evidence that visuospatial processing in the medial temporal lobe is right lateralized.” (Bird, Capponi, King, Doeller, \& Burgess, 2010). Solution of the puzzle: Imaginary scenes are Kantian noumena, left brain inner sense entities, while external objects produce images in the right hemisphere, being Kantian phenomena. Boundaries of imagined scenes therefore clearly fall under the left-lateral inner sense: It is noteworthy that the authors appear to expect right-lateral brain activation (wrongly), ignoring that the imagined scene is perceived by Kant's inner sense.

The philosopher Andrew Brook, a top-line expert on Kant, responded to my email, in which I related to him my new Kantian brain model, rejecting my conclusions, saying the neural results could be interpreted in any number of different ways. This objection flies in the face of Darwinian selection principles, according to which organisms evolve by adaptation of their structures and functions to environmental constraints, and do so in the most economical way possible. The bilateral organization according to my model parallels Kant's two senses \& his partition of objects into noumena \& phenomena, as well as Plato's earlier analogous conception of two worlds, because both of these ingenious men were able to discern that the ontological nature of the environment requires us to be created, or as we would say now, evolve with dual senses and coordinated distinction of temporal inner sense noumena logic vs. spatial outer sense phenomena geometry. In fact, Plato's insights similarly seem to anticipate Darwinian—right brain—adaptation to his sensible world \&—left brain - to his intelligible world as natural metaphysical adaptive constraints.

My question for set theory, does this matter and how would we partition Platonist vs. Formalist thinking between the two brain halves?

I probably should amplify the question somewhat, briefly: Apparently, Plato's detailed theory of perception as he told it in the allegories of the Cave and the Divided Line, was really an ingenious anticipation of the bilateral brain, he put the inner cosmos of the left brain into the heavens that for us today are a place of chaotic matter in galactic clusters \& black holes. Later, Kant came along and placed Plato's heaven into the mind, but did even better anticipating details of neuroscience in his Critiques. 


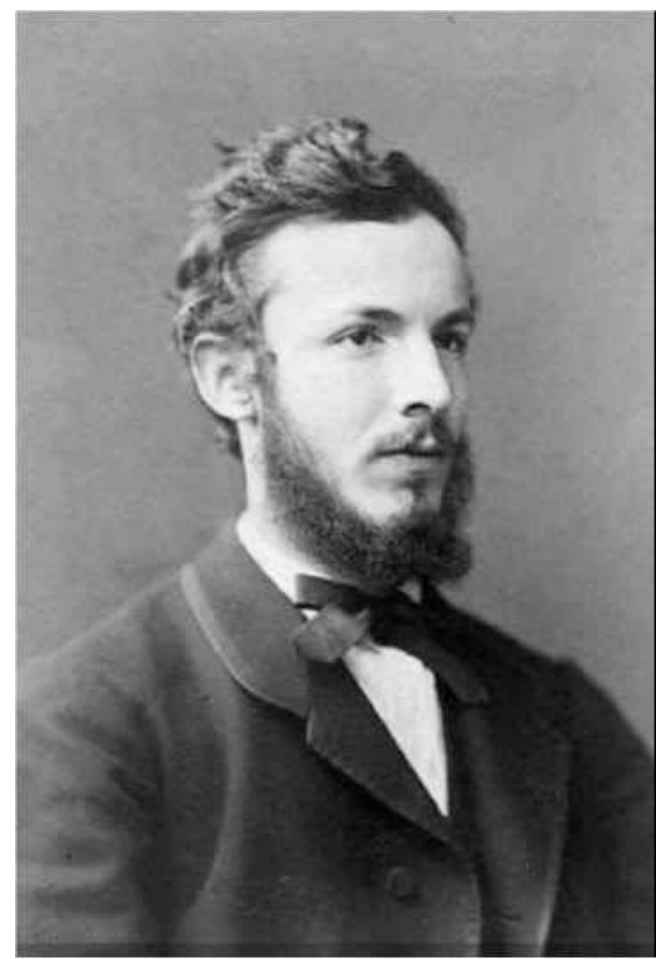

Figure 1. Georg Cantor. Mathematical Institute, Univ. Hamburg.
A major part of the answer is to locate abstract sets in the logical world of our left brain inner, noumenal cosmos, with strong roots in the geometry of the phenomenal cosmos of outer sense objects in the right cerebral hemisphere. The bilateral inner cosmos so divided, probably can be identified with the Default Mode Network (DMN) discovered by Marcus Raichle, WUSTL (Raichle, Macleod, Snyder, Powers, Gusnard, \& Shulman, 2001). This refers to the frontal lobe part of DMN. The parietal lobe is somewhat separate, the conceptual categorical apparatus (CCA) or toolbox. Coincidentally, Raichle too was awarded the 2014 Kavli prize, sharing it with Brenda Milner (and a 3rd scientist). Holistic principles demand that all of our epistemology is worked out by fitting new data into the existing noumenal cosmos and constantly reorganizing the whole, as the DMN works ceaselessly 24/7.

This is known to physiological psychologists as a P300 event related potential (ERP) seen on the EEG. It is a positive voltage observed on the EEG $300 \mathrm{~ms}$ after a novel "odd-ball” stimulus.

From gymnasium days I can recall my first time in the Roman Pantheon, the impression was overwhelming. But by next day, it already had been fitted into my inner cosmos, neuro-anatomically the DMN, \& had turned into the familiar same old...!!

Heidegger has this fitting process covered in his 1935 metaphysics lectures (available in two English translations from MIT Press). This now is called top-down (TD) processing, important vitally for object identification to all animals, because bottom-up (BU) is hopelessly inefficient. As one neuroscientist put it, in some of the half-dozen sequential visual processing centers $\mathrm{V} 1, \mathrm{~V} 2, \ldots$, there are more fibers running backward—for TD processing feed-back—than forward.

That humans possess such an inner cosmos is a tremendous benefit. As the complete ordered experience of the individual, it forms the foundation of judgment and of a sound mind. Treasured by our forebears as their "precious soul," one's inner cosmos must be guarded against deleterious influences, which can lead to mental and even physical illnesses (Burchard, 2005, 2011). ${ }^{1}$

Would mathematical philosophers be able to agree that these recently discovered brain facts do seem to matter to set theory and to set-theorists. And, what if anything should be the common sense understanding of working mathematicians concerning sets, classes \& the monster class $\Omega$ in particular...??

\footnotetext{
1 An expanded treatment is posted on ResearchGate, unpublished.
} 


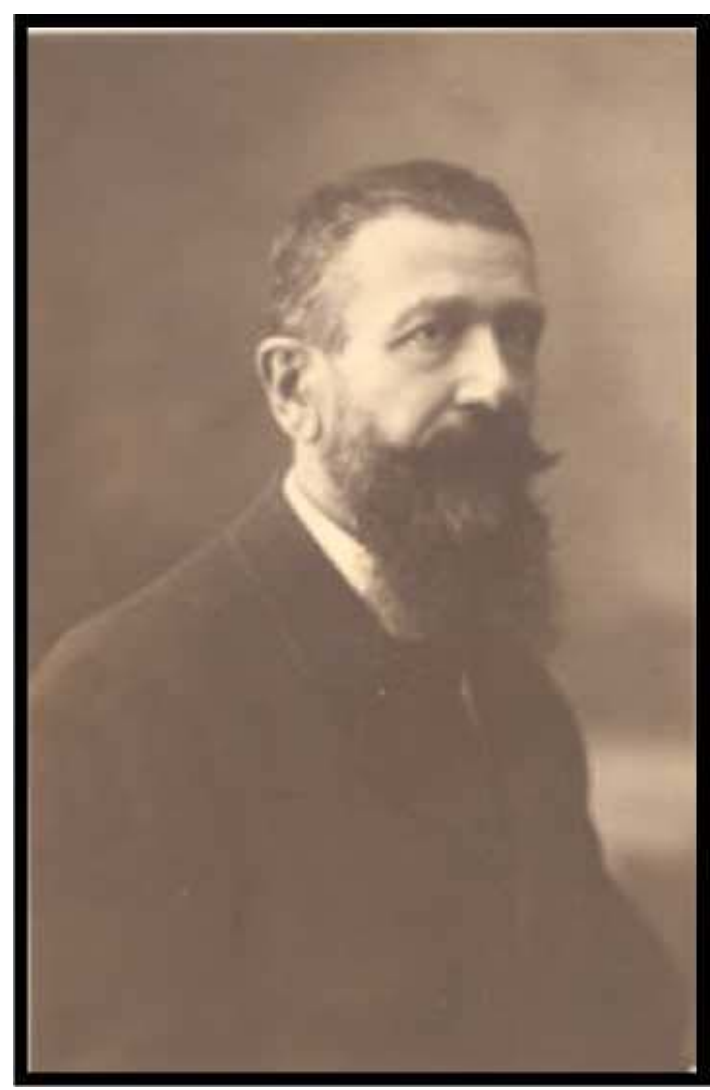

Figure 2. Cesare Burali-Forti. Wikipedia.
As explained above, it should be fairly obvious that abstract mathematical sets are in the left brain, but as the two hemispheres are connected massively through the corpus callosum, there is a great deal of geometry involved in our set theoretic intuitions, esp. the continuum hypothesis (CH). We intuit optically the real line \& are convinced that it has a definite cardinality. This connection through white matter, neural axons, is why the views of the Platonist (right brain spatial geometry) \& Formalist (left brain sequential logic) coexist \& harmonize in our minds.

The Burali-Forti class $\Omega$ is a brain weave connected to reality only in the remotest way. It deserves an examination of how exactly, by which meta-physical or mathematical considerations, we may be able to evaluate or judge the ontological status of this monstrous non-entity, that nonetheless we cannot abrogate for being diffuse, nor can we assuage our discomfort by relegating it to non-sets.

Not directly related to mathematics, a theme of human endeavor opens up, where TD processing, being so prevalent in perception of the momentary scene, confirms long-held suspicions to the extent that apparent reality is merely what we think that it is, what we make it out to be, with a relativistic quantum universe separated from us by the metaphysical gap, as we first learnt, in a primitive analogy, from Plato's cave (Burchard, 2014). Extending this line of thought, sets and the ancestral predicate calculus might be seen as the scaffolding that holds up the building of our experience while it is undergoing construction during our lifetime. The limitless class $\Omega$ crashing through all reasonable bounds would then remind us of the fisherman \& his greedy wife in the Brothers Grimm's fairytale of the magical flounder, taking us back toward our hovel, there humbly to do our calculations \& draw our diagrams.

(1) Given any well ordered set, or any set and a well-order imposed on it, we can think of it as an initial segment of $\Omega$, without explicit mention of $\Omega$.

(2) Given any two well ordered sets, one may be identified as an initial segment or start of the other, and both as starts of $\Omega$.

(3) Given any two well ordered sets, a third one is their sum of which both are starts, as well as of $\Omega$.

(4) Similarly for other binary operations, product or power, a pair generates a third, larger start of $\Omega$.

(5) Talking about ordinals can replace well ordered sets, and all operations may be seen as proceeding inside $\Omega$. 
However, logicians can adopt a more casual point of view, ontological considerations being extraneous to their model theoretic work, where we attempt to understand uncountable ordinals of the highest cardinalities while effectively dealing with countable models. The only inaccessible ordinal likely will turn out to be the one logicians sometimes denote by “Ord," $=\Omega$.

In his publications Cantor never seems to have mentioned $\Omega$ explicitly, although it was certainly implicit in his work. A typical example can be found §III.9.14 on page 321 of his collected works posthumously published by Ernst Zermelo (Cantor, 1932):

Theorem A. If $\alpha$ and $\beta$ are any two ordinals, then either $\alpha=\beta$, or $\alpha<\beta$, or $\alpha>\beta$.

Clearly, when speaking of "any two ordinals" we implicitly are referencing their totality, class $\Omega$.

Two years later, in his letter to Richard Dedekind he draws a careful distinction between sets and classes, calling the latter inconsistent multiplicities (Cantor, 1932).

Suffering from depression, he went on long walks around Friedrichroda in the Harz Mountains, where he often stayed in a health-resort.
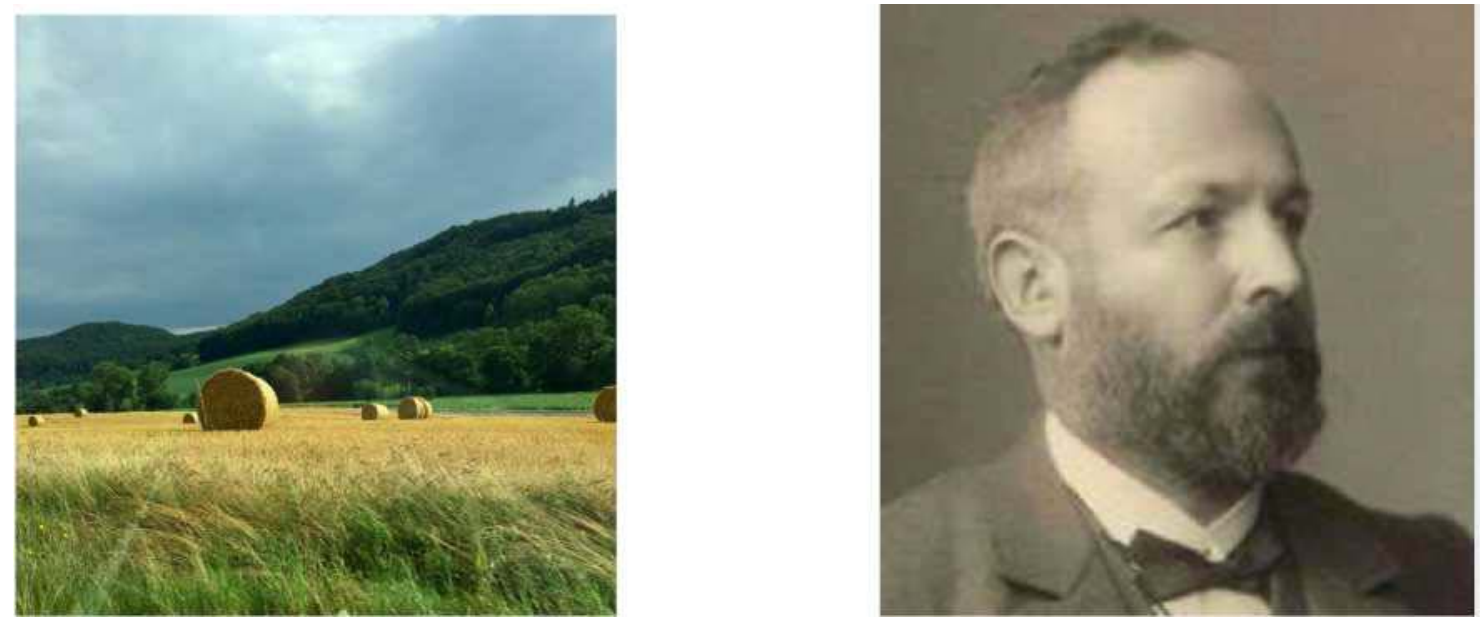

Figure 3. Left: Landscape near Friedrichroda. Melanie Kahl, Fototour Thueringen Right: Georg Cantor. Encyclopdia Britannica.

Cantor, adopting Burali-Forti's choice of the symbol, explains that $\Omega$ is a class not a set and sketches a proof of the Well-Ordering Theorem in the unpublished letter.

If however the totality of elements of a class can be thought of as "being together" without leading to a contradiction, so that its collectedness in "one thing" is possible, then I call it a consistent class or a set.

Obviously, Cantor here is describing a clear case of failure of Kant's Function of Oneness, an entity that we cannot compute in left-brain circuits. Class here appears as another word for Kant's manifold. His training in Kantian thought schemes is becoming evident: ${ }^{2}$

Perhaps some explanation of the oneness function is in order, its neglect in Western philosophy, being that part of my mental activity that generates Kant's celebrated synthesis (Brook, 2018). Inspection of the cerebral

\footnotetext{
${ }^{2}$ Cantor's own terms, more literally translated, for class are multiplicity, system.
} 
architecture, the anatomical location should be the fronto-polar cortex, where operational control is passed from the sensory ventral \& dorsal streams to the motor cortex, the frontal lobe apex being where final decisions are rendered on how to proceed in my existence, the "do or die." Brodmann area BA10 seems to be implicated as the place where my consciousness window opens up on reality, the momentary scene, and my place in it foremost. Neurons in BA10 must have global receptive fields, meaning their synapses firing takes into account everything that I possibly can know, be aware of at that moment, including my own existence, my hopes, my joy, my pain, my plans for the next 30 seconds. Unfortunately however, BA10 includes 200 million neurons, so to understand how this nerve center can produce oneness from the manifold would require someone far more clever than the present author. By sensing ourselves acting we know there must be a self in us, Kant's transcendental Ego is performing his function of oneness. This is his more precise version of the Cartesian "Je pense, donc je suis.” We can crank up the realism of this one more notch: Yeah, BA10's 200 million neurons are forming some kind of non-recursive super-Turing machine by the cells, each one a Turing machine, freely synapsing amongst one another (Burchard, 2016).

A class is called well-ordered, if it satisfies the condition that every partial class has a first element; such a class I call a Folge.

Note that he wishes to allow non-set classes to be well-ordered, in contravention of Burali-Forti's prohibition.

But to our understanding of the nature of non-set classes and of the monster "absolute infinite" class $\Omega$ in particular, we are bound to limit ourselves to subsets of the monstrous non-entity $\Omega$. It is true of course that, allowing the language, every proper initial segment of $\Omega$ would qualify as a well-ordered set, except non-entities should have no initial segments, "nihil ex nihilo."

Also to consider, $\Omega$ should be defined even if only tacitly, given that we know that well-ordered sets are comparable as each other's initial segments.

\section{Adapting Cantor's Sketch for a Transparent Proof of WOT Using $\Omega$}

Our main interest in this essay is in a new, non-controversial proof of WOT based on Cantor's somewhat cryptic remarks using to advantage the paradoxical class $\Omega$ (Cantor, 1899).

To present our new proof of the Well-Ordering Theorem (WOT), which involves some simple but uncommon considerations in set theory, we begin by sketching a bit of the mathematical background from around the start of the 20th century, time period of the widespread adoption of Georg Cantor's set theory, including his powerful transfinite induction based on his discovery, or ingenious invention, of transfinite ordinals (ordinal numbers).

In fact, the proof idea of WOT was suggested by Cantor in a 1899 letter written to Richard Dedekind where he works through Burali-Forti's paradox (Burali-Forti, 1897). The well-known antinomy arises from the class $\Omega$ of all ordinals (ordinal numbers) being an entity that is not itself a set, despite the definition of the predicate "well-ordered set" appearing to be free of any obvious logical errors.

When Georg Cantor published his theory of well-ordered sets, in this early stage of terminological novelty, Cantor showed himself still to have been naive about the distinction between sets and proper classes. ${ }^{3}$

This is evident §III.9.6, page 295 of his collected works (Cantor, 1932):

\footnotetext{
${ }^{3}$ We are accustomed today to keep this in mind, based on NBG \& extended ZFC recursive axiomatics of set theory.
} 
It is to be shown that the transfinite cardinal numbers can be ordered according to magnitude and in this order form a "well-ordered set," like finite numbers do, but in a wider sense. ${ }^{4}$

Curiously, Zermelo, in his note [9] page 352 takes Cantor here to be promising a future program that he never was able to carry out, but we believe that Cantor meant to have delivered on this plan. In fact the proof of WOT that this paper presents, in one form or another, may have been in the back of his mind all along, leading him to be dismissive of the issues, at a time when several prominent authors missed the precise definitions that he had worked out in is papers (Heijenoort, 1967).

Zermelo tries to help out by supplying another item Cantor had passed over, the correct definition of the alephs, note [5] page 352 §III.9 (Cantor, 1932): “And yet it suffices to define the cardinal number in question [meaning its aleph] through the smallest initial segment of a (transfinite) well-ordered set, without a largest element.” The last part of course is redundant.

An equivalent more formal definition of the alephs in terms of injections into $\Omega$ from set $\mathrm{V}$, is given below, Definition B.

The great question had been, whether besides the alephs there are still other potencies of sets; for two years now I have been in possession of a proof that there are no others, so that for example a definite aleph is attributable to the linear continuum-the totality of all real numbers.

We need to have available some terminology related to well-orders. Our point of view will be that we legitimize mathematical proofs by restricting involvement of the class to injections $j$ of sets $V$ into $\Omega, j: V \rightarrow \Omega$, so the range $j(V)$ can be dealt with as a well-ordered set, with its subsets $\Gamma=j(H), H \subset V$.

Definition: An initial segment $\Gamma=j(H), H \subset V$, of the class $\Omega$ is any subset $\Gamma$ such that, if $\mu \in \Omega$ and $\mu<$ $j(m) \in \Gamma$, then $\mu \in \Gamma$.

Definition 0: If $\mu \in \Omega$ then $\Omega(\mu)=\{\alpha \in \Omega: \alpha<\mu\}$, the initial segment of $\mu$.

He then argues for $\Omega$ to be inconsistent, using Burali-Forti's argument, because we could adjoin an additional element to obtain a new Folge $\Omega$ '. If $\Omega$ ' would be consistent, then it would be an ordinal $\mu$ that would be greater than all ordinals in $\Omega$, but we know as a fact that $\Omega$ contains all ordinals, including $\mu$, so $\mu<\mu$, a contradiction. Hence $\Omega$ must be inconsistent likewise.

He next proves, or sets out to prove, that among the alephs $\aleph(\mu)$ of all ordinals $\mu$ there occur the potencies (cardinalities) of all sets. This amounts to a version of the well-ordering theorem. The fact is of great interest as Cantor here is amending himself, whose earlier pronouncement often is quoted, cryptically stating that no proof was needed, as the theorem was a "principle of thought."

Our proof below, which we believe to be much more transparent than standard ones, may shed light on what Cantor really had in mind with his untypical remark, coming from a mathematician whom Zermelo often praises in footnotes for the beauty of his results and proofs. As later work by Zermelo has shown, with follow-ups by Kurt Godel and Paul Cohen, we best get a Zermelo choice function from the axiom of choice, $c f$. below, Definition C.

Definition A: A segment map is an injection $j: V \rightarrow W$ where $W$ is well-ordered and $j(V) \subset W$ is an initial segment of $W$.

\footnotetext{
4 “Es soll gezeigt werden, daß...” signifies imminent demonstration.
} 


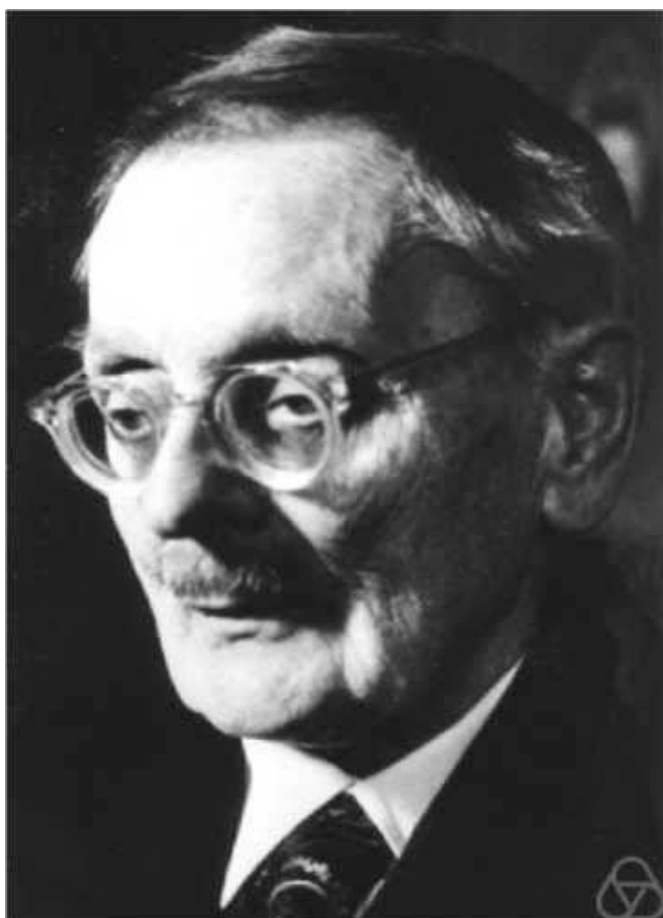

Figure 4. Ernst Zermelo. Univ. Freiburg.

Next, Zermelo's definition of the alephs as stated above, given by him as he complains about Cantor's nonchalant manner of sketching his proof to the effect that alephs are identical to the cardinal numbers.

Definition B: For a set $V$, its aleph, $\mu=\aleph_{r}=$ $|V|$, is defined as the minimal initial segment $\Omega(\mu)$, for which $\exists j, \quad j$ a segment mapping $j: V \rightarrow \Omega(\mu)$.

Note, Zermelo states this without use of symbols, and such injections exist by our WOT proved below. The least $\Omega(\mu)$ automatically will be without a maximal ("last”) element.

Note too, the existence of the least $\mu$ is guaranteed by well-order as long as $\exists j$, a segment map $j$ such that $j: V \rightarrow \Omega$.

Definition C: A Zermelo choice function $\partial$ on a set $V$ satisfies the choice property: If $K \subset V$, then $\partial(K) \in K$, provided $K$ is nonempty.

Definition D: For a given Zermelo choice function $\partial$ on a set $V$, a $\partial$-segment map is any segment map $j$ with domain a subset $F$ of $V, \quad j: F \rightarrow \Omega$ such that

$\forall \mu \in \Omega$, if $\mu \in j(F) \subset \Omega$, then $\mu=j(\partial(V \mid H))$, where $H=j^{-1}(\Omega(\mu))$.

The mysterious proof suggestion Cantor's, p. 447, in edited form. If $V$ is a class $^{5}$ and $|V|$ has no corresponding aleph, then we will conclude, under the premise made, that $V$ must not be a set. For, it is easy to see that we may inject the entire class $\Omega$ into the class $V$, i.e., $\exists \alpha$ subclass $V^{\prime} \subset V$ equivalent to $\Omega$. This shows $V$ is not a set. Therefore, for every transfinite set, there must be a definite aleph as its cardinal number. The key point is labeled "weak" by Zermelo in a note p. 451.

We felt that it would be easier to prove the contrapositive of this argument: If $V$ is a set, then we can produce an injection $j: V \rightarrow \Omega$, hence $|V|=|j(V)|$, but $j(V)$ is an aleph, as defined by Cantor (Zermelo).

Let's analyze this \& begin by restating Cantor's condition using Zermelo's definition. If class $V$ has no corresponding aleph, then $V$ is not a set, a proper class.

We negate Zermelo's definition:

A set $\mathrm{V}$ does not possess an aleph $\gamma \in \Omega$ as its cardinal number iff $\exists j, \quad j$ is a segment mapping, $\& j: V \rightarrow$ $\Omega(\gamma)$.

Note the existence of the least $\gamma$ would be guaranteed by well-order if $\exists j, \quad j$ is a segment map $\& j: V \rightarrow$ $\Omega$.

Notice the discussion reveals that WOT amounts to nothing but every set has an aleph for its cardinal

\footnotetext{
${ }^{5}$ Including sets.
} 
number.

Next, we set out to deliver proof that such injections exist if V is a set, exhuming Cantor's proof of WOT from long slumber in the forest of mathematics. ${ }^{6}$

Essentially, we just are translating Zermelo's 1st proof of WOT (1904). We see this as a reasonable interpretation of Georg Cantor' sketch from his 1899 letter to Dedekind. He there states that he had been in possession of the proof for two years, which would date his proof to the year 1897.

As explained, for a special Zermelo choice function $\partial$ on $V$, we consider $\partial$-segment maps $j$, with domain $F$ a subset of $V$, "client" maps, for short $j: F \rightarrow \Omega, F \subset V$.

The pullback of the well-order of $\Omega$ makes each client $\mathrm{F}$ a well-ordered set, with its smallest element being $m_{1}=\partial(V)$.

For each element $\mu$ in the range of $j$, its initial segment $\Omega(\mu)$ will be contained in the range $j(F)$ of $j, \Omega(\mu)$ $\subset j(F)$.

Begin with $F_{0}=\{\}, F_{1}=\left\{m_{1}\right\} \subset V$, where $m_{1}=\partial(V), V=\bigvee\{\}, j\left(F_{1}\right)=\{1\}$, and $m_{2}=\partial\left(\bigvee\left\{m_{1}\right\}\right), F_{2}=\left\{m_{1}\right.$, $\left.m_{2}\right\}$, with $j_{k}, k=0,1,2$ the counting maps to $\Omega$ and map $m_{1} \mapsto 1, m_{2} \mapsto 2 \in \Omega . \Omega=\{1,2,3, \ldots\}$, then we verify the conditions in the above Definition D.

For any two nonidentical client maps $j: F \rightarrow \Omega, F \subset V$ and $k: G \rightarrow \Omega, G \subset V$, by Definition A, both ranges $j(F)$ and $k(G)$ are initial segments of $\Omega$, so one, say $k(G)$, is an initial segment of the other, $j(F), k(G) \subset$ $j(F) \subset \Omega$. We show that the inverse images of the two $\partial$-segment client maps agree on the smaller range $k(G)$ :

If not, let $\mu \in k(G)$ be the least element for which the inverse images differ under client maps $j \& k$ :

$$
j^{-1}(\mu) \neq k^{-1}(\mu)
$$

But, the initial segment $\Omega(\mu)$ is contained in both ranges, and, by the minimal property of $\mu$, we know that on $\Omega(\mu)$, the inverse images of $j \& k$ agree, hence $k^{-1}(\Omega(\mu))=j^{-1}(\Omega(\mu))=H$, \& therefore by Definition D

$$
j(\partial(V \backslash H))=\mu=k(\partial(V \backslash H)),
$$

hence taking inverse images under each of the two injections,

$$
j^{-1}(\mu)=\partial(V \mid H)=k^{-1}(\mu) .
$$

We have shown they agree, contrary to equation (1).

This proves that any two client maps are extensions of each other.

Thus the maximal 2 -segment map $j$ exists, $j: F \rightarrow \mathrm{Q}, F \subset V$, as union of all client maps. This is a non-controversial, consistent definition of a union of a set of sets.

If $F$ is properly contained in $V$, then we set $m=\partial(V F), \mu=j(m)=j(F)+1$, the successor of the ordinal $j(F)$, by extending the domain $F$ of $j$ to $F \cup\{m\}$, we would have shown $F$ not to be maximal.

Combining what we were able to prove, we can see that we will get all of $V$ for the maximal domain of client maps $j, F=\operatorname{dom}(j)=V$. This completes our proof of the existence of an injection of $V \rightarrow \Omega$, inducing a well-ordering on $V$ by $j$-pullback from the order on class $\Omega$.

\section{References}

Bird, C. M., Capponi, C., King, J. A., Doeller, C. F., \& Burgess, N. (2010). Establishing the boundaries: The hippocampal contribution to imagining scenes. The Journal of Neuroscience, 30, 11688-11695. https://dx.doi.org/10.1523/jneurosci.0723-10.2010

Brook, A. (2018). Kant's view of the mind and consciousness of self. The Stanford Encyclopedia of Philosophy, ed. Edward N.

\footnotetext{
${ }^{6}$ There is lingering doubt whether the proof obtains a minimal $j, \quad j(V)=\Omega(\mu) \& \aleph_{\gamma(\mathrm{V})}=\mu$.
} 
Zalta. https://plato.stanford.edu/archives/win2018/entries/kant-mind

Burali-Forti, C. (1897). Una Question Sur Numeri Transfiniti. Rendiconti del Circolo Matematico di Palermo, 11, $154-164$. https://doi.org/10.1007/BF03015911

Burchard, H. G. W. (2005). Symbolic languages and natural structures. Foundations of Science, 10, $153-245$. https://dx.doi.org/10.1007/s10699-004-3068-9

Burchard, H. G. W. (2011). The role of conscious attention in perception. Foundations of Science, 16, 67-99. https://dx.doi.org/10.1007/s10699-010-9201-z

Burchard, H. G. W. (2014). The cognitive gap, neural Darwinism \& linguistic dualism. Open Journal of Philosophy, 4, $244-264$. Published Online August 2014. https://dx.doi.org/10.4236/Ojpp.2014.43032

Burchard, H. G. W. (2016). Philosophy and science, the Darwinian-evolved computational brain, a non-recursive super-turing machine \& the inner-world- producing organ. Open Journal of Philosophy, 6, 13-28. Published Online February 2016. https://dx.doi.org/10.4236/ojpp.2016.61002

Burchard, H., \& Burchard, J. (2016). Mentoring arithmetic skills for children and adults of all ages using alphabet blocks. In N. Dominguez, and D. Alexander (Eds.), 9th Annual Mentoring Conference Proceedings: Developmental Networks: The Power of Mentoring and Coaching. University of New Mexico, Albuquerque, NM.

Georg Cantor. (1932). Gesammelte Abhandlungen: Mathematischen und philosophischen inhalts. Publisher: Ernst Zermelo Berlin, Heidelberg: Springer Berlin Heidelberg: Imprint: Springer, 1932.

Georg Cantor. (1895). Beitrage zur Begründung der transfiniten Mengenlehre (1. Artikel). Mathematische Annalen, 46, 481-512. https://doi.org/10.1007/BF02124929

Georg Cantor. (1897). Beitrage zur Begründung der transfiniten Mengenlehre, II. Mathematische Annalen, 49, $207-246$. https://doi.org/10.1007/BF01444205

Frisk, V., \& Milner, B. (1990). The role of the left hippocampal region in the acquisition and retention of story content. Neuropsychologia, 28, 349-359. http://www.sciencedirect.com/science/journal/00283932/28/4

Jean van Heijenoort. (1967). From Frege to Godel: A source book in mathematical logic: 1879-1931. Cambridge, MA: Harvard U.P.

Igloi, K., Doeller, C. F., Berthoz, A., Rondi-Reig, L., \& Burgess, N. (2010). Lateralized human hippocampal activity predicts navigation based on sequence or place memory. Proceedings of the National Academy of Sciences of the United States of America, 107, 14466-14471. https://dx.doi.org/10.1073/pnas.1004243107

Kunen, K. (1980). Set theory. An introduction to independence proofs. Studies in Logic and the Foundations of Mathematics, 102. Elsevier Science.

Milner, B. (1971). Interhemispheric differences in the localization of psychological processes in man. British Medical Bulletin, 27, 272-277. http://bmb.oxfordjournals.org/content/by/year/1971

Raichle, M. E., Macleod, A. M., Snyder, A. Z., Powers, W. J., Gusnard, D. A., \& Shulman, G. L. (2001). A default mode of brain function. Proceedings of the National Academy of Sciences of the United States of America, 98, 676-682. https://dx.doi.org/10.1073/pnas.98.2.676

Smith, M. L., \& Milner, B. (1989). Right hippocampal impairment in the recall of spatial location: Encoding deficit or rapid forgetting? Neuropsychologia, 27, 1-81. http://www.sciencedirect.com/science/journal/00283932/28/4

Ernst Zermelo. (1904). Beweis, daß jede Menge wohlgeordnet werden kann. Mathematische Annalen, 59, S.514-516. https://doi.org/10.1007/BF01445300

Ernst Zermelo. (1908). Neuer Beweis für die Möglichkeit einer Wohlordnung. Mathematische Annalen, 65, S.107-128. https://doi.org/10.1007/BF01450054 